\title{
Dispersed-Phase Structure of Pressure-Atomized Sprays at Various Gas Densities
}

\author{
L. K. Tseng, ${ }^{*}$ P. K. Wu, ${ }^{*}$ and G. M. Faeth $\dagger$ \\ University of Michigan, Ann Arbor, Michigan 48109
}

\begin{abstract}
The dispersed-phase structure of the dense-spray region of pressure-atomized sprays was studied for atomization breakup conditions, considering large-scale $(9.5 \mathrm{~mm}$ initial diameter) water jets in still-air at ambient pressures of 1,2 , and $4 \mathrm{~atm}$, with both fully developed turbulent pipe flow and nonturbulent slug flow at the jet exit. Drop sizes and velocities and liquid-volume fractions and fluxes were measured using holography. Measurements were compared with predictions based on the locally homogeneous flow (LHF) approximation as well as recent correlations of drop sizes after primary breakup of turbulent and nonturbulent liquids. The dispersed-flow region beyond the liquid surface was relatively dilute (liquid-volume fractions less than $0.1 \%$ ), with significant separated-flow effects throughout, and evidence of near-limit secondary breakup and drop deformation near the liquid surface. Turbulent primary breakup predictions were satisfactory at atmospheric pressure, where the correlation was developed, but failed to predict observed trends of decreasing drop sizes with increasing gas density due to aerodynamic effects; in contrast, the laminar primary breakup predictions successfully treated the relatively small effects of gas density for this breakup mechanism. Effects of liquid turbulence at the jet exit were qualitatively similar to single-phase flows, yielding faster mixing rates with increased turbulence levels even though drop sizes tended to increase as well. LHF predictions within the dispersed-flow region were only qualitatively correct due to significant separated-flow effects, but tended to improve as the ambient pressure and the distance from the jet exit increased.
\end{abstract}

\section{Nomenclature}

$C$
$C$
$d$
$d$
$e_{p}$
$f$
$g$
$L$
$N$

$C_{a} \quad=$ aerodynamic breakup constant, Eq. (7)

$=$ ligament residence-time constant, Eq. (6)

$=$ injector diameter

$d_{p} \quad=$ drop diameter

$e_{p} \quad=$ volume-averaged ellipticity

$f \quad=$ mixture fraction

$g \quad=$ liquid flux

$L \quad=$ injector passage length

$N=$ number of drops in sample

$O h_{i}=$ Ohnesorge number based on length scale $i$, $\mu_{f} /\left(\rho_{f} i \sigma\right)^{1 / 2}$

$R e_{d} \quad=$ jet Reynolds number, $u_{0} d / \nu_{f}$

$r \quad=$ radial distance

$u \quad=$ streamwise velocity

$u_{p} \quad=$ streamwise drop velocity

$V \quad=$ sample volume

$v \quad=$ radial velocity

$W e_{f \Lambda}=$ integral-scale Weber number, $\rho_{f} u_{0}^{2} \Lambda / \sigma$

$\tilde{W} e_{g} \quad=$ spray Weber number, Eq. (2)

$W e_{g p} \quad=$ drop Weber number, Eq. (3)

$W e_{g p}^{*}=$ critical-drop Weber number for secondary breakup

$W e_{g S M D}=$ primary breakup Weber number, $\rho_{g} u_{0}^{2} \mathrm{SMD} / \sigma$

$W e_{i d}=$ jet Weber number based on phase $i, \rho_{i} u_{0}^{2} d / \sigma$

$x=$ streamwise distance

$\alpha \quad=$ liquid-volume fraction

$\Lambda \quad=$ radial integral scale

$\mu \quad=$ molecular viscosity

$\nu \quad=$ kinematic viscosity

$\rho \quad=$ density

Received Sept. 9, 1991; presented as Paper 92-0230 at the AIAA 30th Aerospace Sciences Meeting, Reno, NV, Jan. 6-9, 1992; revision received April 8, 1992; accepted for publication April 28, 1992. Copyright (c) 1992 by the American Institute of Aeronautics and Astronautics, Inc. All rights reserved.

${ }^{*}$ Graduate Assistant, Department of Aerospace Engineering.

†Professor, Department of Aerospace Engineering. Fellow AIAA.

$$
\begin{aligned}
& \sigma \quad=\text { surface tension } \\
& \tau_{b}=\text { ligament aerodynamic breakup time, Eq. (7) } \\
& \tau_{r} \quad=\text { ligament residence time, Eq. (6) } \\
& f \quad=\text { liquid-phase property } \\
& f c \quad=\text { liquid core property } \\
& g \quad=\text { gas-phase property } \\
& 0=\text { injector exit condition }
\end{aligned}
$$

\section{Introduction}

T HE present study further investigates past work in this laboratory concerning the properties of the near-injector dense-spray region of pressure-atomized sprays. ${ }^{1-6}$ The first phase of the investigation involved measurements of liquidvolume fractions and dispersed-phase structure of large water jets ( 9.5 and $19.1 \mathrm{~mm}$ initial diam) in still-air at atmospheric pressure. ${ }^{1-3}$ Tseng et al. ${ }^{4}$ extended the liquid-volume fraction measurements to treat effects of ambient air density at ambient pressures of 1-8 atm. Additionally, mechanisms of primary breakup of both nonturbulent and turbulent liquids have been studied in air at normal temperature and pressure while considering various liquids and jet-exit diameters and velocities. ${ }^{56}$ The objective of the present investigation was to examine the effect of varying gas densities on the dispersedphase properties of dense sprays, emphasizing conditions within the multiphase mixing layer that begins right at the jet exit for atomization breakup conditions, for test conditions similar to Tseng et al. ${ }^{4}$

Past work on pressure-atomized dense sprays has been discussed in several recent articles. ${ }^{7-12}$ Early studies emphasized breakup regimes, identifying conditions for Rayleigh, windinduced and atomization breakup - the latter having greatest practical importance because of its wide range of operating conditions and production of small drops needed for rapid mixing. ${ }^{11-15}$ Subsequent work concentrated on visualization of the near-injector region of the flow and definition of the 
properties of the liquid core, which is much like the potential core of a single-phase jet. ${ }^{15-20}$ More recently, the properties of the multiphase mixing layer surrounding the liquid core have been studied, beginning with measurements within the dilute spray region near the outer edge of this flow. ${ }^{21,22}$

Past work in this laboratory has largely concentrated on the properties of the near-injector region during atomization breakup, using relatively large diameter liquid jets (3.5-19.1 $\mathrm{mm}$ ) to provide known jet-exit flow conditions and reasonable spatial resolution because the flow diameter is not thought to affect dense spray structure appreciably. ${ }^{1-6}$ This has included measurements of liquid-volume fraction distributions using gamma-ray absorption for water jets in air at various ambient pressures. ${ }^{1,4}$ These results showed that mixing rates were influenced by the breakup regime, turbulence properties at the jet exit, and the ambient gas density. Predictions based on the locally-homogeneous flow (LHF) approximation, where relative velocities between the phases are neglected, were in good agreement with measurements for Favre-averaged mixture fractions greater than 0.85 , but overestimated mixing rates at lower mixture fractions. The reasons for this behavior were explored by measuring the structure of the multiphase mixing layer at atmospheric pressure-drop sizes, phase velocities, entrainment rates, and liquid-volume fractions and fluxes-using holography and phase-discriminating laser velocimetry. ${ }^{2,3}$ The results showed significant effects of separated flow so that good performance of the LHF approximation only occurred at relatively large mixture fractions where the small velocities of the gas and small drops were not very important.

The most recent work in this laboratory has considered the properties of primary breakup, using holography to find drop sizes and velocities at atmospheric pressure. ${ }^{1.5}$ Two modes of primary breakup were considered: 1) turbulent breakup, due to distortion of the liquid surface by turbulence; and 2) nonturbulent breakup, due to stripping of liquid from boundary layers formed on the windward side of waves along the liquid surface. Correlations of Sauter mean diameter (SMD) after primary breakup were achieved for both modes of breakup for a variety of liquids and jet-exit diameters and velocities, but have only been evaluated for liquid jets in air at atmospheric pressure.

The present investigation extends the earlier work in this laboratory, ${ }^{1-6}$ addressing effects of ambient gas density on the properties of the dispersed phase during atomization breakup by observing water jets in still-air at pressures of 1,2 , and 4 atm with both turbulent and nonturbulent jet-exit conditions. Holography was used to find drop sizes and velocities, and liquid-volume fractions and fluxes, within the dispersed-flow region beyond the liquid surface. Following earlier work, ${ }^{1-4}$ predictions of flow properties using the LHF approximation were used to help assess separated-flow effects on the structure of the flow. The correlations for primary breakup properties were also evaluated for effects of varying ambient gas density. The present discussion is brief; additional details and a complete tabulation of data can be found in Tseng. ${ }^{23}$

\section{Experimental Methods}

\section{Apparatus}

The test apparatus involved a steady water jet injected vertically downward within a large-windowed pressure vessel $(1.5-\mathrm{m}$ diam $\times 4.5-\mathrm{m}$ long $){ }^{+}$The rate of water flow was adjusted with a bypass system and measured using a paddle wheel flowmeter. Two injectors having exit diameters of 9.5 $\mathrm{mm}$ were used: 1) one yielding slug flow with low-turbulence intensities; and 2) the other yielding fully developed turbulent pipe flow. The slug-flow injector consisted of a honeycomb flow straightener, two screens to calm the flow and a 13.6:1 area contraction designed following Smith and Wang ${ }^{2+}$ to yield uniform velocities at the exit. The fully developed flow injector had a similar configuration with the contraction fol- lowed by a constant area passage 41 jet-exit-diam long to yield nearly fully developed turbulent pipe flow at the exit. The injectors could be traversed to accomodate the rigidly mounted holocamera.

\section{Holocamera}

The holocamera and reconstruction systems were the same as Ruff et al. ${ }^{2}$ Measurements were obtained over $6 \times 6 \times$ $4-\mathrm{mm}^{3}$ volumes, using at least three holograms per position. The data was spatially averaged over the width of the measuring volumes, or $\pm \frac{1}{2}$ the distance between adjacent radial positions, whichever was smaller. Drops and other nearly spherical objects were sized by finding the maximum and minimum diameters through the centroid of the image. Assuming that the object was ellipsoidal, its diameter was taken to be the diameter of a sphere having the same volume as the ellipsoid. This procedure was not appropriate for elongated liquid elements where the centroid was outside the boundaries of the image. Then, the projected area and perimeter of the image were measured and the maximum and minimum diameters of the ellipsoid having the same cross-sectional area and perimeter were computed to find the effective sphere diameter as before. The ellipticity, defined as the ratio of the maximum and minimum ellipsoid diameters, was also computed for each object.

Drop-velocity measurements were based on the motion of the centroid of the image and were correlated as a function of diameter using a least-squares fit. This allowed plots of drop velocities for fixed-drop diameters across the width of the mixing layer while making maximum use of the data on the holograms at each position. Knowing the volume and velocities of liquid elements in the flow, liquid-volume fractions and fluxes could be computed in a straightforward manner.

Measurements typically involved analysis of 150 objects at each position and pressure. Experimental uncertainties generally were dominated by sampling limitations rather than resolution of the reconstructed holograms. Experimental uncertainties ( $25 \%$ confidence) were as follows ${ }^{23}: \mathrm{SMD}<10 \%$, $e_{p}<15 \%$, liquid object velocities $<20 \%, \alpha<15 \%$, and $g$ $<25 \%$. All measurements were repeatable well within these limits.

\section{Test Conditions}

Mean flow conditions were the same for the slug and fully developed flows and were identical to Tseng et al. ${ }^{4}$ This involved a water-flow rate of $3.47 \mathrm{~kg} / \mathrm{s}$, an average jet-exit velocity of $49.1 \mathrm{~m} / \mathrm{s}, R e_{d l}=462,000, O h_{d}=0.00121, W e_{f d l}=$ 312,000 , and $W e_{g d}=380,760$ and 1520 at pressures of 1,2 , and $4 \mathrm{~atm}$. Due to limitations of the pump, this water-flow rate was roughly $13 \%$ lower than the atomization breakup condition considered by Ruff et al. ${ }^{1-3}$ for the same injectors. However, present flows were well within the atomization breakup regime defined in Refs. 13 and 14.

Ruff et al. ${ }^{1}$ completed laser velocimeter measurements at the jet exit for the present injectors, spanning the present operating condition. For slug flow, mean streamwise velocities were uniform over the central region and declined near the wall (within $3-5 \%$ of the injector radius), due to boundary-layer growth in the nozzle passage, while rms velocity fluctuations were roughly $1 \%$ of the mean streamwise velocity over the central portion of the flow. For fully developed flow, mean velocity distributions were in good agreement with literature values for the same Reynolds number range, while rms velocity fluctuations near the axis were somewhat larger than literature values..$^{25.26}$

\section{Theoretical Methods}

Predictions of flow properties were limited to the use of the LHF approximation similar to past work. ${ }^{1-4} \mathrm{~A}$ detailed description of the approach is provided elsewhere. ${ }^{7.23}$ In addition to the LHF approximation, the major assumptions of 
the model are as follows: steady (in the mean) axisymmetric flow with no swirl, boundary-layer approximations apply, negligible kinetic energy and viscous dissipation of the mean flow, buoyancy only affects the mean flow, equal exchange coefficients of all species and phases, and negligible mass transport between the phases (no evaporation). The formulation followed the conserved-scalar formalism of Lockwood and Naguib $^{27}$ but used Favre averages following Bilger. ${ }^{28}$ The specific formulation, all empirical constants, calibration of the approach for variable density single-phase flows, the numerical computations, and specification of initial conditions are described in Refs. 1,4 , and 7.

\section{Results and Discussion}

\section{Drop Properties}

Similar to earlier findings, ${ }^{3,5,6}$ present measurements of dropsize distributions correlated quite well with Simmons' universal root-normal distribution function. ${ }^{29}$ The root-normal distribution is defined by two moments: the SMD and the ratio of the mass median diameter (MMD) to the SMD. Typical of past observations, ${ }^{3,5,6,29} \mathrm{MMD} / \mathrm{SMD}=1.2$ for present turbulent and nonturbulent flows so that the entire drop-size distribution will be represented by the SMD in the following.

Typical distributions of drop properties across the mixing layer for fully developed flow (illustrating flow properties for turbulent primary breakup) appear in Figs. 1-4; see Tseng ${ }^{23}$ for similar plots at all test conditions. Figures $1-3$ illustrate flow properties at pressures of 1,2 , and 4 atm at the fixed streamwise position of $x / d=6$. Figures 3 and 4 illustrate effects of increasing $x / d$ from 6 to 25 at a fixed pressure of 4 atm. The plots include $e_{p}, \mathrm{SMD}$, and drop velocities for $d_{p}$ $=10,50,100$, and $200 \mu \mathrm{m}$. The range of positions where the surface of the liquid core was observed and LHF predictions of flow velocities are also shown on the plots.

Many of the features of volume-averaged ellipticity and SMD seen in Figs. 1-4 are similar to earlier observations of Ruff et at. ${ }^{2}$ at atmospheric pressure; however, significant effects of increasing ambient pressure are observed as well.

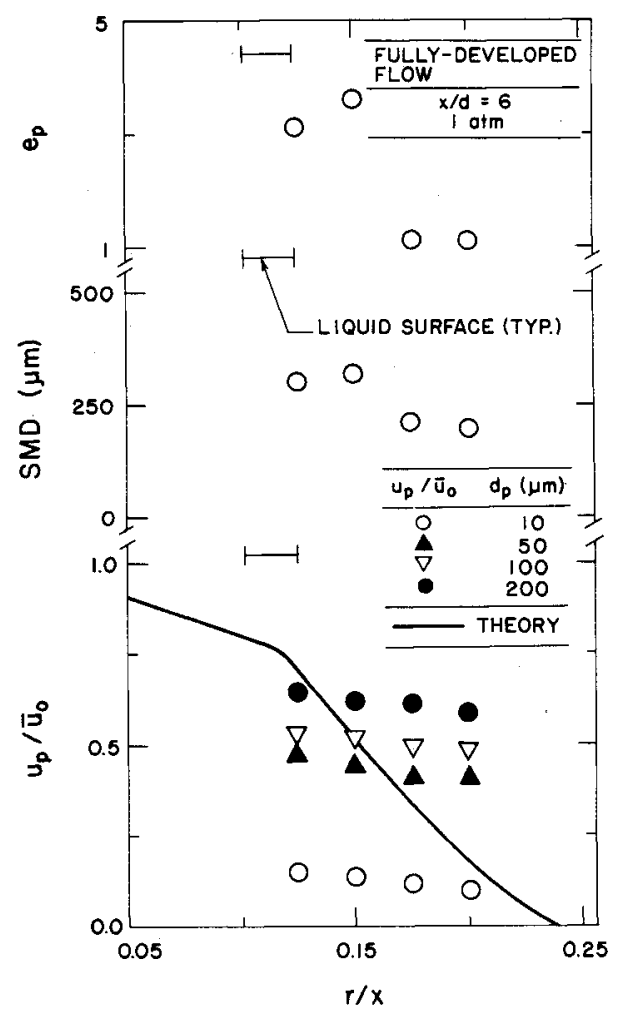

Fig. 1 Dispersed-phase properties for fully developed flow at $x / d=$ 6 and $1 \mathrm{~atm}$.

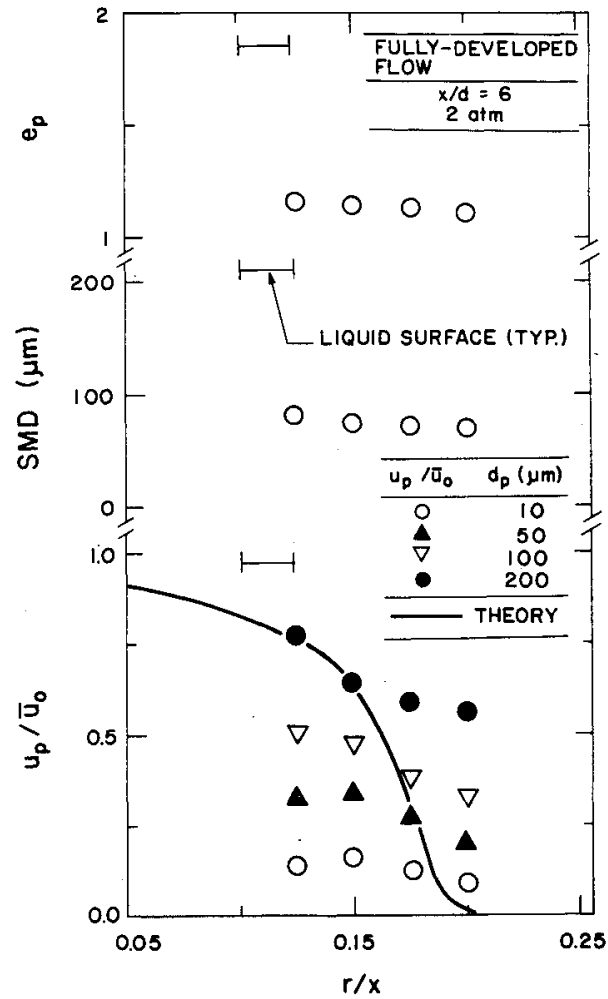

Fig. 2 Dispersed-phase properties for fully developed flow at $x / d=$ 6 and 2 atm.

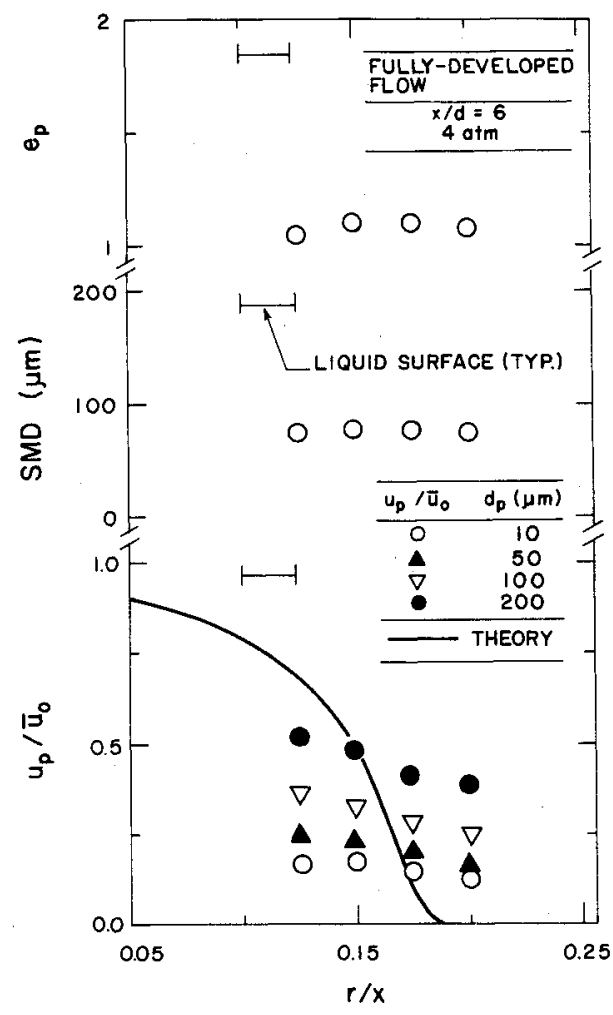

Fig. 3 Dispersed-phase properties for fully developed flow at $x / d=$ 6 and 4 atm.

Thus, at atmospheric pressure, the largest values of $e_{p}$ and SMD generally are in the region of the liquid surface and with increased radial distance $e_{p}$ approaches unity, and the SMD decreases, representing smaller round drops near the edge the flow. This behavior supports the presence of primary breakup yielding large drops which subsequently undergo secondary breakup. With increasing pressure at $x / d=6$, how- 


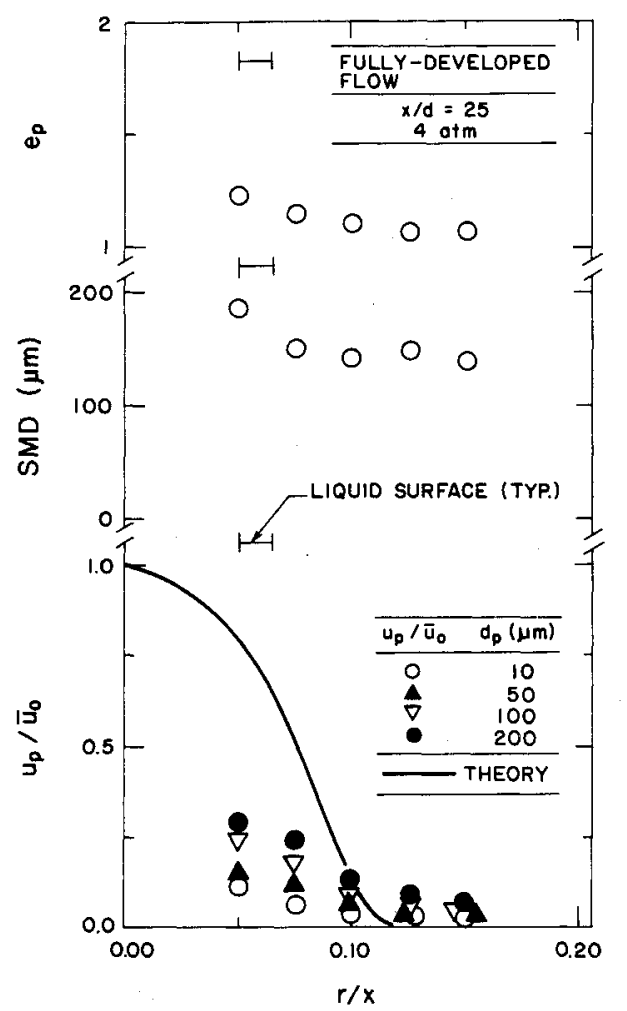

Fig. 4 Dispersed-phase properties for fully developed flow at $x / d=$ 25 and 4 atm.

ever, $e_{p}$ remains near unity and the SMD is relatively uniform across the flow. This reflects the increased propensity for secondary breakup, and shorter secondary breakup times, for particular drop sizes and relative velocities, as the gas density increases. ${ }^{3}$ This altered behavior at increased gas densities is also evident from the substantial reduction of SMD near the surface as the ambient pressure increases. This suggests the merging of primary and secondary breakup processes as the ambient pressure increases. Effects of streamwise distance on $e_{p}$ and SMD seen at atmospheric pressure ${ }^{2,6}$ are still preserved at elevated pressures for present test conditions. Thus, comparing results at $x / d=6$ (Fig. 3) and 25 (Fig. 4) shows increasing $e_{p}$ and SMD with increasing distance from the jet exit that is characteristic of turbulent primary breakup. ${ }^{6}$ Smaller relative velocities, caused by faster mixing rates at elevated pressures, also are probably a factor in this behavior.

Distributions of drop velocities in Figs. 1-4 have been normalized by the mass-averaged velocity at the exit of the jet. In general, there are substantial differences between the velocities of drops of different size, with drop velocities generally overestimated near the liquid core and underestimated near the edge of the flow by the LHF predictions. This is clear evidence of significant separated flow effects. Drop velocities tend to decrease with increasing radial distance (reflecting effects of longer residence times in the flow for drops farther from the surface) and reduced drop diameters (reflecting smaller velocity relaxation times of smaller drops). Increasing pressure and $x / d$ tend to decrease velocities for particular drop sizes near the edge of the flow. This is due to reduced velocity relaxation times with increased ambient pressures for the Reynolds numbers typical of drops in sprays. Drops near the surface are less affected by $x / d$ increases, however, because they tend to be recently formed and have velocities representative of the liquid core which do not change significantly over the range of present observations. ${ }^{4}$ A surprising feature of the velocity measurements is that the velocities of small drops (which provide a reasonable upper bound for gas velocities) remain low and relatively uniform across the flow, particularly at atmospheric pressure and small $x / d$ (see Fig. 1). This implies that momentum exchange between the liquid and gas is not very effective. Such behavior is caused by the relatively large velocity relaxation times of large drops which contain most of the liquid momentum.

\section{Mean-Phase Velocities}

The LHF predictions yielded mass-weighted (Favre)-averaged velocities; therefore, present measurements were used to compute these velocities, similar to Ruff et al. ${ }^{4}$ To do this, mean-gas velocities were taken to be equal to the mean velocities of $5-\mu \mathrm{m}$-diam drops, which represents the smallest drop size that could be resolved during the holography measurements. Naturally, velocities of drops of this size only represent a potential upper-bound for gas velocities because their velocity relaxation times are too large for them to be accurate seeding particles to find gas velocities. However, this effect is not very significant because the large drops contain most of the momentum of the flow. Favre-averaged velocities were found by summing over $V$ containing $N$ drops as follows:

$$
\tilde{u}=\frac{\sum_{i=1}^{N} \rho_{f} d_{p i}^{3} u_{p i}+\left(6 V / \pi-\sum_{i=1}^{N} d_{p i}^{3}\right) \rho_{g} \bar{u}_{g}}{\sum_{i=1}^{N} \rho_{f} d_{p i}^{3}+\left(6 V / \pi-\sum_{i=1}^{N} d_{p i}^{3}\right) \rho_{g}}
$$

The evaluation of Eq. (1) only involved the dispersed-flow region where drops had separated from the liquid surface. This underestimates the true Favre-averaged velocity near the liquid surface because contributions from the liquid core and attached ligaments are ignored.

Mean phase velocities for fully developed and slug-flow jetexit conditions are illustrated in Figs. 5-8. Three mean velocities are shown: 1) $\tilde{u}$ found from Eq. (1);2) $\bar{u}_{g}$ taken to be the mean time-averaged velocities of drops having a diameter of $5 \mu \mathrm{m}$; and 3) the LHF predictions. Two predictions are illustrated for slug flow to indicate potential effects of the boundary layer forming along the walls of the injector passage: 1) one for $L / d=0$ where the presence of the boundary layer is ignored; and 2) the other allowing for boundary-layer development for a length $L / d=5$ following Schlichting, ${ }^{25}$ see

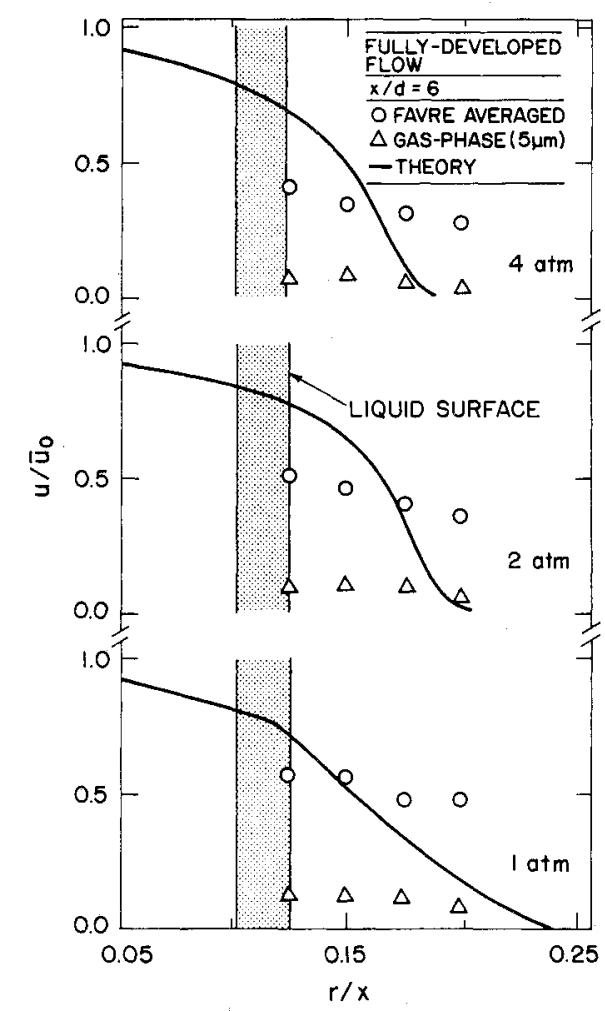

Fig. 5 Mean-phase velocities for fully developed flow at $x / d=6$. 


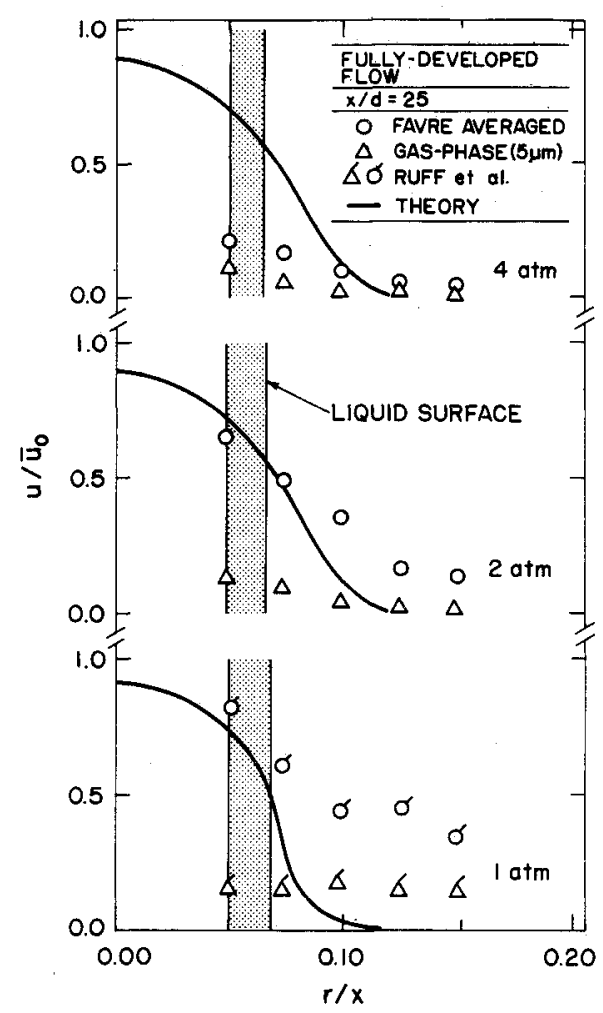

Fig. 6 Mean-phase velocities for fully developed flow at $x / d=25$.

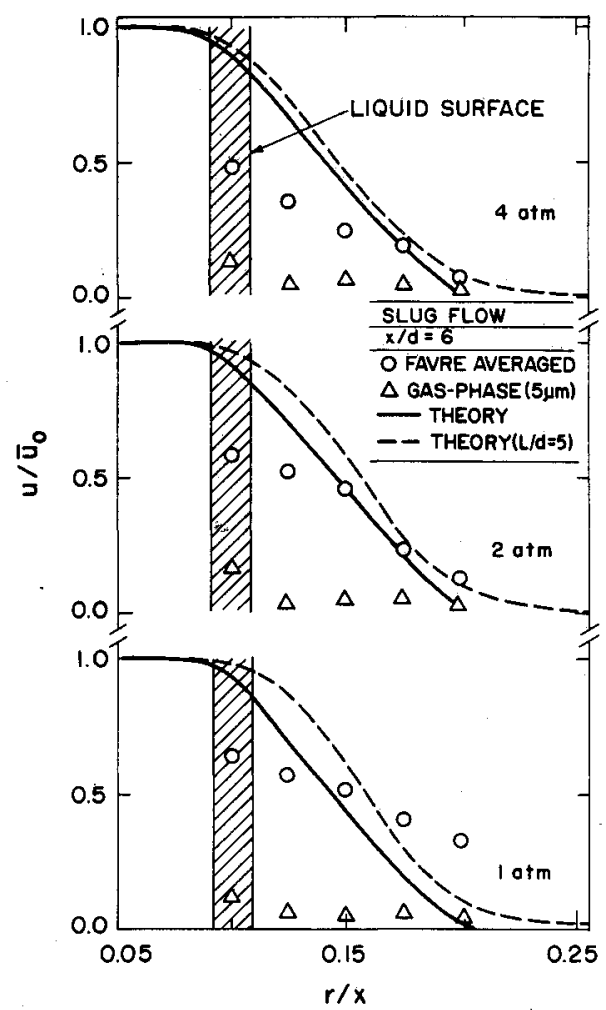

Fig. 7 Mean-phase velocities for slug flow at $x / d=6$.

Refs. 1 and 4 for descriptions of the latter predictions. These conditions bound the range of possibilities for the present slug-flow injector. ${ }^{1}$

Except for two conditions which may not be representative (Fig. 6 at $4 \mathrm{~atm}$ and Fig. 8 at $1 \mathrm{~atm}$ ), measured values of $\tilde{u}$ generally are significantly larger than $\tilde{u}_{g}$ near the liquid surface, with differences between these velocities tending to decrease with increasing radial distance, ambient pressure, and $x / d$. Thus, the dense-spray region near the liquid surface is

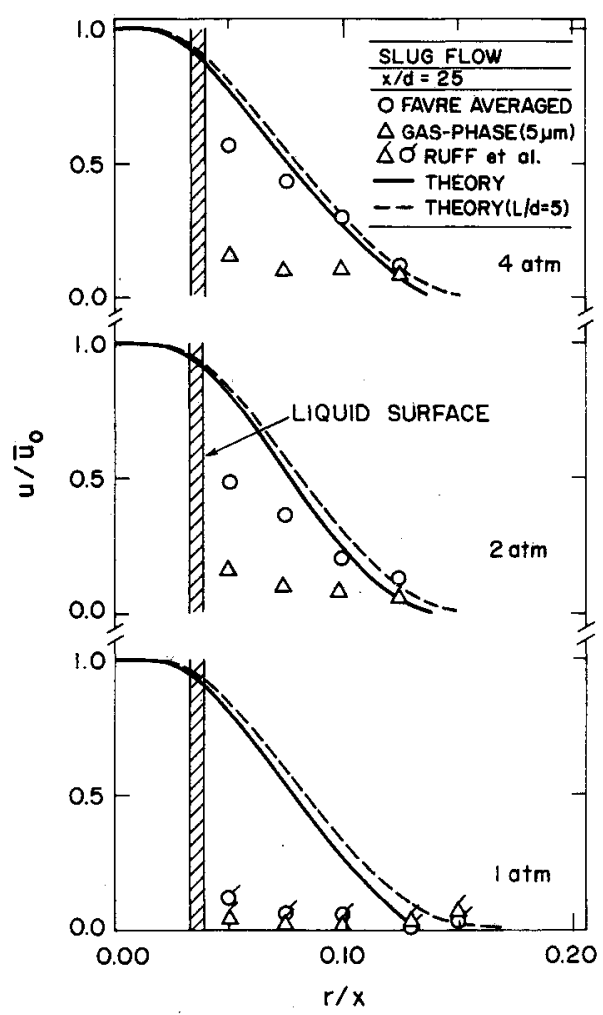

Fig. 8 Mean-phase velocities for slug flow at $x / d=25$.

characterized by large relative velocities that help promote primary and secondary breakup.

The LHF predictions of $\tilde{u}$ are generally not very satisfactory in Figs. 5-8, because present measurements emphasize nearinjector conditions where effects of separated flow remain important. Nevertheless, they tend to improve with increasing distance from the jet-exit and ambient pressure, in accord with earlier scaling arguments. ${ }^{3}$ The tendency toward better agreement of LHF predictions comes about because large drops generally dominate the momentum content of the flow, similar to behavior at larger liquid-volume fractions where the liquid core is present, $, 2,4$ The small drops and the gas all have velocities that are generally much smaller than $\tilde{u}$. In fact, some of the apparent improvement of LHF predictions near the edge of the flow is due to the method of plotting Figs. 58 because all velocities become small so that differences between them are not very apparent. Similar to the findings of Ruff et al. $^{3}$ at atmospheric pressure, Favre-averaged separated flow factors, $\left(\bar{u}_{f}-\tilde{u}_{g}\right) / \tilde{u}_{f}$, generally have values greater than 0.6 for the range of present measurements. ${ }^{23}$ Finally, there is a tendency for LHF predictions to be better for slug than for fully developed flow, (aside from the two atypical conditions noted earlier) because drops are generally smaller and have smaller velocity relaxation times for nonturbulent than turbulent primary breakup..$^{2,5.6}$

The extent of the region where the liquid core is observed is another feature of interest seen in Figs. 5-8. In general, the width of this region does not vary appreciably at a fixed $x / d$ as the ambient pressure varies. However, the effect of liquid turbulence on the liquid core is appreciable, with turbulent jet-exit conditions causing the surface of the liquid to move over a wider range of radial positions and to penetrate farther from the flow axis at a given streamwise position. This behavior is due to distortion (flapping) of the liquid core by the large-scale features of the turbulence which tends to promote mixing, just like the smaller features of the turbulence tend to promote primary breakup. The fact that these characteristics are dominated by jet-exit conditions, rather than aerodynamic effects, is supported by the relatively small effect of ambient pressure on liquid-surface properties. 


\section{Secondary Breakup}

Similar to Ruff et al., ${ }^{3}$ the propensity for secondary breakup in the mixing layer was assessed by computing mass-averaged Weber numbers. Rather than summing over the entire dropsize distribution similar to Ref. 3, however, $\tilde{W} e_{\mathrm{g}}$ was found from the measured values of phase velocities and SMD as follows:

$$
\tilde{W} e_{g}=\rho_{g}\left(\tilde{u}_{f}-\tilde{u}_{g}\right)^{2} \mathrm{SMD} / \sigma
$$

This definition has the advantage that maximum values of individual drop-Weber numbers

$$
W e_{g p}=\rho_{g} d_{p}\left(u_{p}-\bar{u}_{g}\right)^{2} / \sigma
$$

are related quite simply to $\tilde{W} e_{g}$. In particular, $\tilde{u}_{f}-\tilde{u}_{g}$ is characteristic of the relative velocities of the largest drops, while maximum drop diameters are 3-4 times larger than the SMD for the universal-root normal-size distribution function. Therefore, maximum $W e_{g p}$ are roughly 3-4 times larger than $\tilde{W} e_{g}$, whereas more than $50 \%$ of the mass of the spray have $W e_{g p}$ larger than $\tilde{W} e_{g}$.

Criteria for secondary breakup largely depend on dropWeber numbers for drops having low Ohnesorge numbers and large $\rho_{f} / \rho_{g}$, representative of present test conditions. ${ }^{3,10}$ One criterion for water drops accelerated by shock waves is ${ }^{10,30,31}$

$$
W e_{g p}>W e_{g p}^{*}=6.5-12
$$

Slower disturbance rates for water drops falling in air also yield $W e_{g p}^{*}$ on the order of 10 for water drops in air. ${ }^{3,32}$ Finally, $W e_{g p}$ on the order of unity indicates the onset of conditions where drops deform from a spherical shape after shock-wave disturbances at low Ohnesorge numbers and large $\rho_{f} / \rho_{g} \cdot{ }^{31}$

Distributions of $\tilde{W} e_{g}$ are plotted as a function of $r / x$, with ambient pressure and jet-exit conditions as parameters (see Figs. 9 and 10). Most of the drops at the liquid surface exceed the criterion for deformation, but drops near the edge of the

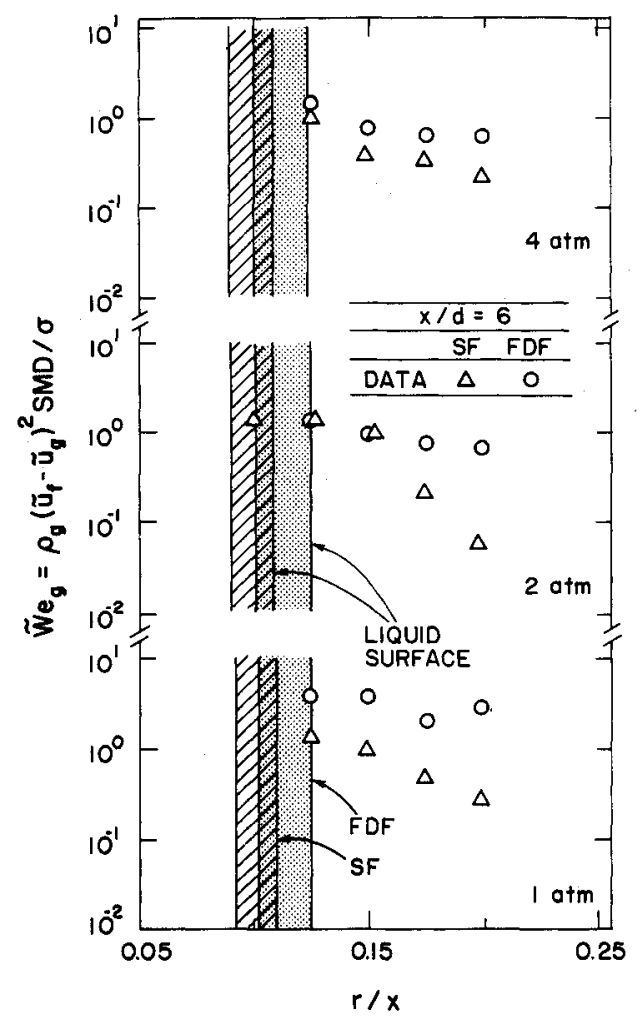

Fig. 9 Favre-averaged drop Weber numbers for fully developed and slug flow at $x / d=6$.

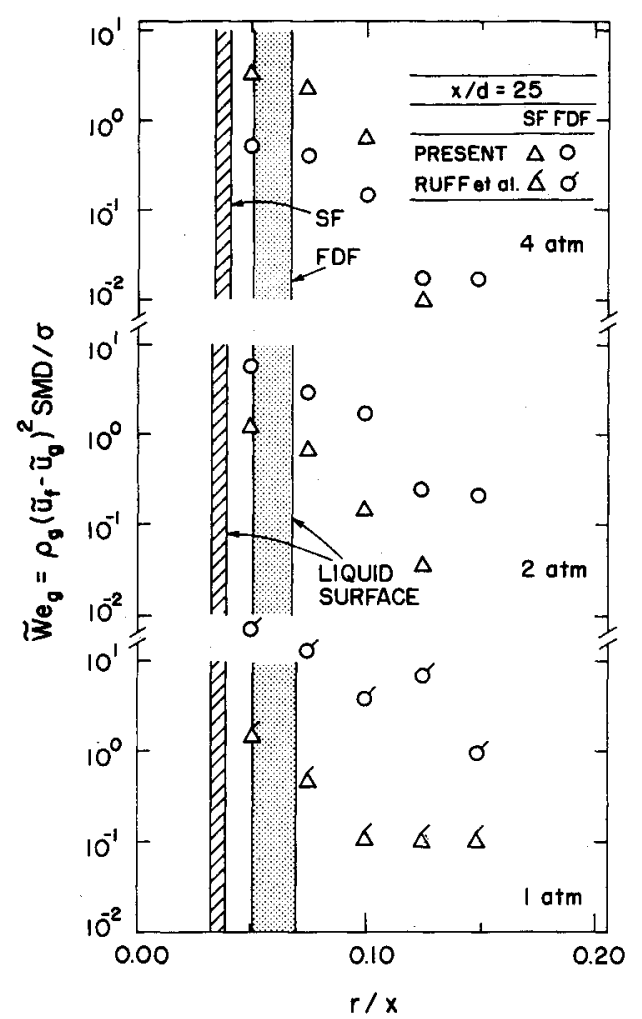

Fig. 10 Favre-averaged drop Weber numbers for fully developed and slug flow at $x / d=25$.

flow are well below this criterion. Direct observations of drop shapes from hologram reconstructions agree with behavior anticipated from the deformation criterion: deformed drops were quite prevalent near the liquid surface while drops near the edge of the flow were generally spherical. This behavior contributes to decreasing values of $e_{p}$ with radial distance, approaching unity near the edge of the flow, although the presence of irregular ligament-like drops contributes to large values of $e_{p}$ near the surface as well.

Results illustrated in Figs. 9 and $\mathbf{1 0}$ also show significant potential for near-limit secondary breakup near the liquid surface for some conditions. In particular, all conditions at atmospheric pressure, as well as all conditions at $x / d=25$, involve significant fractions of drops that are unstable to secondary breakup near the liquid surface. However, drops near the edge of the flow generally have $W e_{g p}$ that are lower than the breakup criterion of Eq. (4). This supports the hypothesis that reduced SMD with increasing radial distance in Figs. 14 is caused by secondary breakup.

Effects of jet-exit conditions also can be seen from the results illustrated in Figs. 9 and 10. Turbulent primary breakup generally yields larger drops than nonturbulent primary breakup, while the velocities of the largest drops near the liquid surface tend to approach liquid-core velocities which are essentially the same in both cases (see Figs. 5-8). Therefore, $\tilde{W} e_{g}$ are largest near the liquid surface, with the greatest propensity for secondary breakup for fully developed jet-exit conditions (aside from the untypical behavior at $x / d=25$ and $4 \mathrm{~atm}$, mentioned earlier). The tendency for processes of primary and secondary breakup to merge at elevated pressures for fully developed flow is also evident from the corresponding reduction of $\tilde{W} e_{g}$ at the liquid surface that is particularly noticeable for fully developed flow. This effect will be discussed in greater detail in the next section.

\section{Primary Breakup}

\section{Turbulent Primary Breakup}

Recent studies have yielded correlations of drop sizes after turbulent and nonturbulent primary breakup..$^{5,6}$ These results 
were developed from measurements for a variety of liquids injected into the air at atmospheric pressure; therefore, it is of interest to evaluate them for potential effects of varying ambient gas density using the present measurements. This will be done in the following, beginning with turbulent breakup.

Wu et al. ${ }^{6}$ developed an expression for the SMD after turbulent primary breakup, ignoring aerodynamic effects. The correlation is based on the projection of liquid elements from the surface by radial velocity fluctuations, relating element size and velocity to the turbulence spectrum within the inertial region. The size of elements leaving the surface is taken to be the largest turbulent scale that can complete its growth at a particular point, with the length of the element determined by Rayleigh breakup considerations. The maximum size produced by this mechanism is taken to be proportional to the integral scale. For second wind-induced and atomization breakup conditions and $O h<0.006$, the resulting expression for the SMD after primary breakup is

$$
\mathrm{SMD} / \Lambda=0.69\left[\left(x /\left(\Lambda W e_{f \Lambda}^{0.54}\right)\right]^{0.57}\right.
$$

where $\Lambda$ is the cross-stream integral scale of the flow. Notably, this expression only involves properties of the liquid phase because aerodynamic effects have been ignored.

Present measurements of SMD along the liquid surface for fully developed flow, along with the predictions of Eq. (5), are summarized in the upper part of Table 1. The agreement between predictions and measurements at atmospheric pressure corresponding to the range of $\rho_{f} / \rho_{g}$ used to develop Eq. (5) is excellent. However, the predictions do not anticipate the substantial reduction of drop sizes after primary breakup as the ambient pressure increases. The progressive reduction of SMD with increasing pressure clearly suggests the presence of aerodynamic effects that are not considered in the phenomenological theory used to develop Eq. (5).

A curious feature of the failure of Eq. (5) to correlate SMD after primary breakup at pressures of 2 and 4 atm is that these expressions were developed over a wide range of $W e_{g d}$, with resulting wide variations of aerodynamic forces; thus, the problem largely appears to be related to the variation of density ratio. Insight concerning this behavior can be obtained by considering the residence times of a typical ligament breaking away from the surface and secondary breakup times for liquid elements of comparable size, using the integral-scale limit to simplify the discussion. Taking the length of the ligament at the time it separates to be $e_{p} \Lambda$, and the velocity of liquid flow into the ligament to be $\bar{v}_{f}^{\prime}$, the characteristic residence time of the ligament during its formation is

$$
\tau_{r}=C_{r} e_{p} \Lambda / \bar{v}_{f}^{\prime}
$$

where $C_{r}$ is a constant on the order of unity. The mode of aerodynamic breakup of the ligaments is not known; fortunately, breakup times are relatively independent of mode. ${ }^{33,34}$ Adopting the correlation of Ranger and Nicholls ${ }^{34}$ for shear breakup, and taking the characteristic ligament dimension to be $\Lambda$, yields the characteristic aerodynamic breakup time of the ligament as follows:

$$
\tau_{b}=5 C_{a} \Lambda\left(\rho_{f} / \rho_{g}\right)^{1 / 2} / u_{0}
$$

where $C_{a}$ is an empirical constant of order-unity to allow for shape differences between drops and ligaments, and the relative velocity has been taken to be $u_{0}$, which is representative of velocity differences between ligaments extending from the surface and the local gas velocity. Solving for the ratio of Eqs. (7) and (6) then yields

$$
\tau_{b} / \tau_{r}=\left(5 C_{a} / C_{r} e_{p}\right)\left(\rho_{f} / \rho_{g}\right)^{1 / 2}\left(\bar{v}_{f}^{\prime} / u_{0}\right)
$$

Noting that turbulence properties within the liquid do not change appreciably with position, $\bar{v}_{f}^{\prime} / u_{0}$ is simply the intensity of radial velocity fluctuations at the jet exit which is a constant for the fully developed turbulent pipe flow. Thus, Eq. (8) indicates that the relative importance of aerodynamic breakup effects only depends on the density ratio, in agreement with the present measurements and the observations of Ref. 6.

Equation (8) also suggests that the transition from turbulent breakup due to liquid turbulence alone-to conditions where aerodynamic effects also are important-occurs at pressures on the order of atmospheric pressure. For example, $e_{p}$ generally is in the range of $2-4$ at the liquid surface for turbulent breakup (see Ref. 2 and Figs. 1-4); therefore, it is reasonable to take the first factor on the right side of Eq. (8) to be of order-unity. The radial turbulence intensity $\bar{v}_{f}^{\prime} / u_{0}$, for fully developed turbulent pipe flow, generally is in the range $0.03-$ 0.05 .25 .26 Then, at ambient pressures of 1,2 , and $4 \mathrm{~atm}$ for water and air; $\rho_{f} / \rho_{g}=846,423$, and 212 , yielding $\tau_{b} / \tau_{r}=1.2$, 0.8 , and 0.6 (taking the radial turbulence intensity to be 0.04 ). These results suggest that aerodynamic effects begin to become important at ambient pressures on the order of $1 \mathrm{~atm}$ and that this transition is largely a function of density ratio, which agrees with the SMD data of Table 1 . More study is needed to refine these order of magnitude considerations, however, present findings clearly show that the use of Eq. (5) to find SMD for $\rho_{f} / \rho_{g}$ less than the range considered in Ref. 6 is not appropriate.

\section{Nonturbulent Primary Breakup}

$\mathrm{Wu}$ et al. ${ }^{5}$ have developed an expression giving the SMD after primary breakup for slug flow at the jet exit. This expression is based on aerodynamic stripping of boundary layers formed on the windward-side of waves along the liquid surface. For liquid Reynolds numbers based on distance from the jet exit greater than $2 \times 10^{6}$ (which corresponds to present conditions) a fully developed breakup regime is entered where the SMD after primary breakup is relatively independent of distance from the jet exit. Within this regime, the correlation for SMD along the surface is

$$
W e_{g_{\mathrm{SMD}}}=16\left(W e_{g d} / \operatorname{Re}_{d}^{1 / 2}\right)^{0.82}
$$

\begin{tabular}{|c|c|c|c|c|c|c|}
\hline \multirow[b]{3}{*}{$x / d$} & \multicolumn{6}{|c|}{ Pressure, atm } \\
\hline & \multicolumn{2}{|l|}{1} & \multicolumn{2}{|l|}{2} & \multicolumn{2}{|l|}{4} \\
\hline & Measurement & Prediction & Measurement & Prediction & Measurement & Prediction \\
\hline \multicolumn{7}{|c|}{ Fully developed flow ${ }^{b}$} \\
\hline 6 & 343 & 374 & 133 & 374 & 70 & 374 \\
\hline 25 & 940 & 968 & 343 & 968 & 157 & 968 \\
\hline \multicolumn{7}{|l|}{ Slug flow ${ }^{\mathrm{c}}$} \\
\hline 6 & 117 & 241 & 100 & 213 & 98 & 188 \\
\hline 25 & $143^{\mathrm{d}}$ & $215^{d}$ & 140 & 213 & 138 & 188 \\
\hline
\end{tabular}

where the Weber and Reynolds numbers are based on mean jet-exit velocity, assuming small variations of velocity within

Table 1 Measured and predicted SMD, $\mu \mathrm{m}$, along liquid surface ${ }^{\mathrm{a}}$

"Momentum-averaged injection velocity, $\mathrm{m} / \mathrm{s},{ }^{b}=40, " s=48$, and $d=56$. 
the liquid core and small gas velocities along the surface (the latter generally corresponding to present observations and those of Ref. 3).

Present measurements of SMD along the liquid surface for slug flow, along with the predictions of Eq. (9), are summarized in the lower part of Table 1 . In this case, measured SMD are affected only slightly with increasing pressure and distance along the surface; trends that are consistent with the predictions of Eq. (9) as well as effects of distance within the fully developed nonturbulent breakup regime observed in Ref. 5. However, the predictions overestimate the measured SMD by roughly a factor of two, which is larger than the scatter of the data used to develop Eq. (9). ${ }^{5}$ Specific reasons for this deficiency are not known, however, processes of nonturbulent breakup are notoriously sensitive to small disturbances within the injector so that differences in the injector passage design may be a factor. In any event, unlike turbulent primary breakup, present observations of the trends of nonturbulent primary breakup with variations of $\rho_{f} / \rho_{g}$ appear to be consistent with the observations of Ref. 5. It should be noted, however, that parameters controlling secondary breakup [see Eqs. (4) and (7)] respond more rapidly to increased gas densities than those controlling nonturbulent primary breakup [see Eq. (9)]. Therefore, higher pressures than are considered here may involve the merging of primary and secondary breakup, which is similar to present observations of turbulent primary breakup: this possibility should be explored.

\section{Liquid-Volume Fractions and Fluxes}

Measured and predicted distributions of liquid-volume fractions and fluxes are illustrated in Figs. 11 and 12 for both fully developed and slug flow. Measurements of liquid-volume fractions include both present findings using holography, and those of Tseng et al. ${ }^{4}$ using gamma-ray absorption for the same flows. LHF predictions for slug flow are shown at the limits $L / d=0$ and 5 as before.

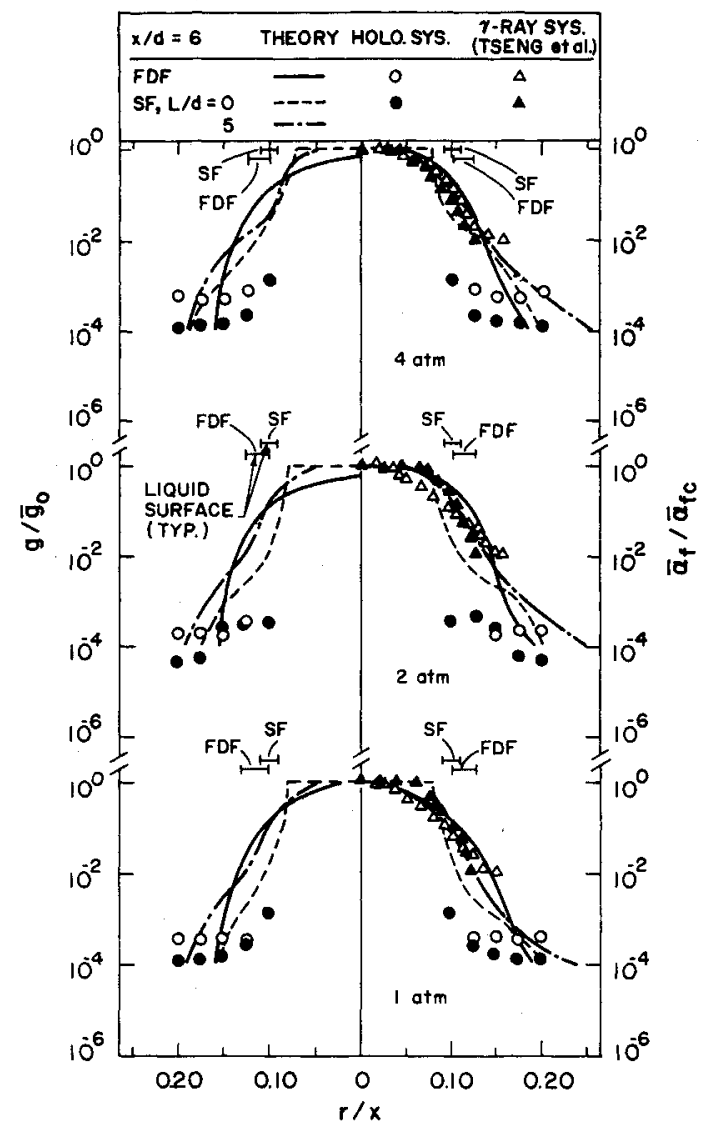

Fig. 11 Liquid volume fractions and fluxes for fully developed and slug flow at $x / d=6$.

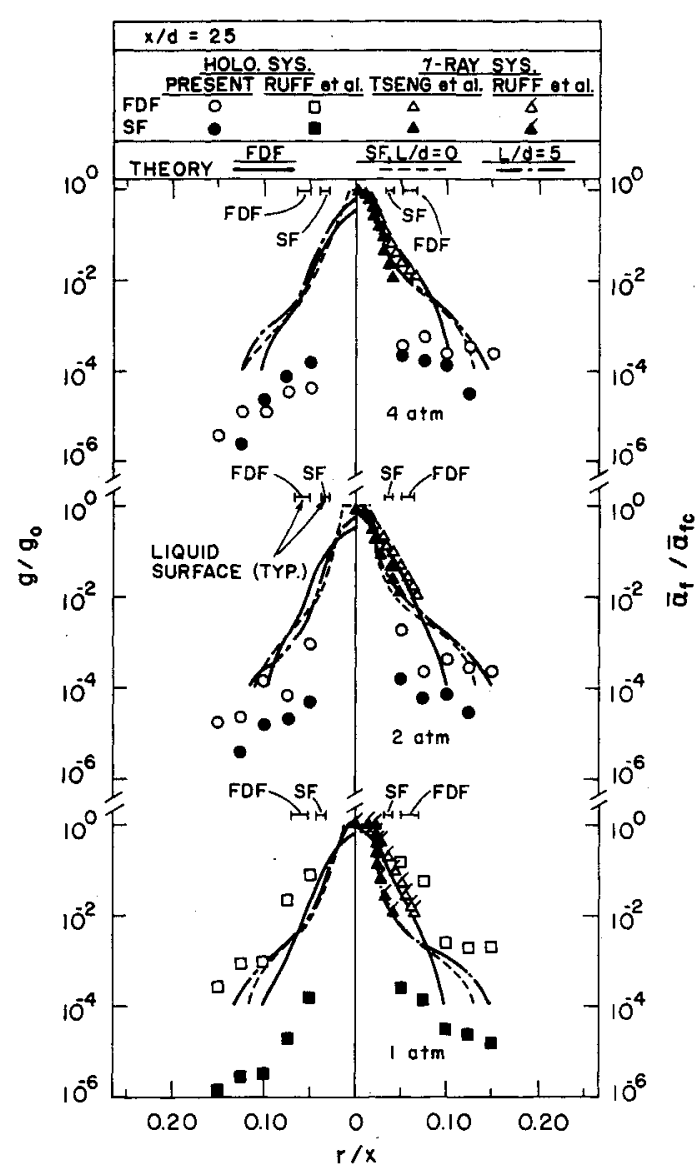

Fig. 12 Liquid volume fractions and fluxes for fully developed and slug flow at $x / d=25$.

The measurements of liquid-volume fractions by gammaray absorption are larger than those found by holography in the region where they overlap in Figs. 11 and 12. This region is associated with the presence of the liquid core; therefore, the main reason for the differences is that the holography measurements do not include the significant contributions of the liquid core and the attached ligaments protruding from its surface. Thus, the discrepancy largely reflects reduced liquid-volume fractions anticipated when moving from a liquid region to a fully dispersed multiphase flow region. Liquidvolume fractions in the dispersed-flow region don't vary appreciably with increasing pressure and distance from the jet exit, with maximum values remaining at roughly $0.1 \%$ where effects of collisions are not very significant. ${ }^{7}$ Distributions of liquid-volume fractions generally are broader, with higher liquid-volume fractions in the dispersed-flow region, for fully developed flow in comparison to slug flow, reflecting the faster mixing rates of the turbulent breakup process at the liquid surface. The LHF predictions of liquid-volume fractions are reasonably good up to the outer edge of the region where the liquid surface is present, which involves liquid-volume fractions greater than 0.1 . Thereafter, the LHF predictions only bear a crude resemblance to measured properties in the dispersed-flow region: in part because the measurements ignore the presence of the liquid surface and attached ligaments, as noted earlier; and because of the significant effects of separated flow in the dispersed-flow region.

The liquid-flux measurements and predictions illustrated in Figs. 11 and 12 are qualitatively similar to the liquid-volume fractions. Fluxes for fully developed flow generally are higher than for slug flow due to the faster rates of turbulent primary breakup (except for the untypical behavior for fully developed flow at $x / d=25$ and 4 atm, Fig. 12, noted earlier). Measurements of liquid fluxes do not account for contributions from the liquid core and its attached ligaments and under- 
estimate fluxes in the region of the liquid surface as a result. Accounting for this, the LHF predictions are in qualitative agreement with the measurements, although consideration of separated-flow effects is needed to develop a quantitatively accurate methodology.

\section{Conclusions}

The near-injector region of pressure-atomized water sprays ( $9.5 \mathrm{~mm}$ initial diameter) was investigated at ambient air densities corresponding to ambient pressures of 1-4 atm, considering atomization breakup conditions for both fully developed and slug flow at the jet exit. The main conclusions of the study are as follows:

1) Similar to earlier observations at atmospheric pressure, ${ }^{2,3.5 .6}$ drop sizes at each point satisfied Simmons' universal root-normal drop-size distribution, ${ }^{29}$ with an MMD/SMD ratio of 1.2. Thus, the entire drop-size distribution can be characterized by a single moment, like the SMD.

2) Drop sizes after primary breakup generally were larger for fully developed than for slug-flow jet-exit conditions, highlighting the importance of injector passage disturbances on spray properties. The correlation of Wu et al. ${ }^{6}$ for SMD after turbulent primary breakup was in excellent agreement with present measurements at atmospheric pressure (the same ambient pressure used to develop the correlation). However, the correlation did not represent measured trends of reduced SMD after primary breakup as the ambient pressure increased. This suggests the presence of aerodynamic effects, or merging of primary and secondary breakup that were not considered when the correlation was developed. The difficulty is largely associated with an effect of density ratio; therefore, the correlation of Ref. 6 should only be used for $\rho_{f} \rho_{g}>580$, which was the range originally used to define it.

3) Measured trends of effects of ambient pressure and distance from the injector on SMD after nonturbulent primary breakup were in good agreement with the correlation developed by $\mathrm{Wu}$ et al. ${ }^{5}$ which includes consideration of aerodynamic effects, although present values were $50 \%$ smaller than predicted. This implies relatively small effects of ambient pressure on drop sizes after nonturbulent primary breakup so that merging of primary and secondary breakup at higher pressures may still be a factor, due to the larger response of secondary breakup processes to changes in gas density. The size discrepancy and the potential merging effect require additional study before the correlation of Ref. 5 can be applied confidently to high-pressure sprays.

4) Spray Weber numbers after primary breakup imply that a significant fraction of the drops will undergo near-limit secondary breakup (particularly at low pressures and far from the injector) while most of the drops exceed conditions where they deform from a spherical shape, even if they do not undergo secondary breakup. Drops near the edge of the flow, however, have Weber numbers well below values associated with secondary breakup and deformation.

5) Similar to earlier observations at atmospheric pressure, ${ }^{2}$ liquid-volume fractions in the dispersed-flow region beyond the liquid surface were relatively low, less than $0.1 \%$. Thus, the flow in this region corresponds to a dilute spray, aside from added complications of secondary breakup and irregular or deformed drops.

6) Favre-averaged separated flow factors $\left(\tilde{u}_{f}-\tilde{u}_{g}\right) / \tilde{u}_{f}$ were generally greater than 0.6 throughout the dispersed flow region, implying significant effects of separated flow and surprisingly low gas velocities even near the liquid surface. These effects suggest that turbulence generation by drops, ${ }^{7,8}$ is a major feature of the dispersed flow region.

7) Predictions based on the LHF approximation were only qualitatively useful due to the significant separated-flow effects in the dispersed-flow region. Performance of the LHF approach tended to improve with increasing liquid-volume fractions, ambient pressure, and distance from the injector.
Pending resolution of existing uncertainties about the properties of primary and secondary breakup, the LHF approach offers a useful treatment of sprays in the atomization breakup regime at high ambient pressures where drop sizes are likely to be small. It should be recognized, however, that the LHF approach will overestimate the rate of development of the flow by a degree that cannot be quantified until uncertainties about breakup and other properties of the near-injector region are resolved.

\section{Acknowledgments}

This research was sponsored by the Office of Naval Research Grant N00014-89-J-1199 under the technical management of G. D. Roy; initial apparatus development was sponsored by the Air Force Office of Scientific Research, Grant 89-0516 with J. M. Tishkoff serving as technical manager. The U.S. Government is authorized to reproduce and distribute copies for governmental purposes. notwithstanding any copyright notation thereon.

\section{References}

${ }^{1}$ Ruff, G. A., Sagar, A. D., and Faeth, G. M., "Structure of the Near-Injector Region of Pressure-Atomized Sprays," AIAA Journal, Vol. 27, No. 7, 1989, pp. 901-908.

${ }^{2}$ Ruff, G. A., Bernal, L. P., and Faeth, G. M., "Structure of the Near-Injector Region of Non-Evaporating Pressure-Atomized Sprays," Journal of Propulsion and Power, Vol. 7, No. 2, 1991, pp. 221-230.

${ }^{3}$ Ruff, G. A., Wu, P.-K., Bernal, L. P., and Faeth, G. M., "Continuous- and Dispersed-Phase Structure of Dense Nonevaporating Pressure-Atomized Sprays," Journal of Propulsion and Power, Vol. 8, No. 2, 1992, pp. 280-289.

${ }^{4}$ Tseng, L.-K., Ruff, G. A., and Faeth, G. M., "Effects of Gas Density on the Structure of Liquid Jets in Still Gases," AIAA Journal, Vol. 30, No. 6, 1992, pp. 1537-1544

${ }^{s}$ Wu, P.-K., Ruff, G. A., and Faeth, G. M., "Primary Breakup in Liquid/Gas Mixing Layers," Automization and Sprays, Vol. 1, No. 4, 1991, pp. 421-440

${ }^{6}$ Wu, P.-K., Tseng, L.-K., and Faeth, G. M., "Primary Breakup of Turbulent Liquids," AIAA Paper 92-0462, Reno, NV, Jan. 1992.

'Faeth, G. M., "Mixing, Transport and Combustion in Sprays," Progress in Energy and Combustion Science, Vol. 13, No. 3, 1987, pp. 293-345.

${ }^{8}$ Faeth, G. M., "Structure and Atomization Properties of Dense Turbulent Sprays," Twenty-Third Symposium (International) on Combustion, Combustion Inst., Pittsburgh, PA, 1990, pp. 1345-1352.

${ }^{9}$ Chigier, N. A., "The Physics at Atomization," Proceedings of the Fifth International Conference on Liquid Atomization and Spray Systems, National Inst. of Standards and Technology, NIST SP-813, Washington, DC, July 1991, pp. 1-15.

${ }^{10} \mathrm{Clift}$, R., Grace, J. R., and Weber, M. E., Bubbles, Drops and Particles, Academic Press, New York, 1978, p. 346

"Reitz, R. D., and Bracco, F. V., "Mechanism of Atomization of a Liquid Jet," Physics of Fluids, Vol. 25, No. 10, 1982, pp. 17301742 .

${ }^{12}$ Reitz, R. D., "Atomization and Other Breakup Regimes of a Liquid Jet," Ph.D. Dissertation 1375-T, Princeton Univ., Princeton, NJ, 1978.

${ }^{13}$ Miesse, C. C., "Correlation of Experimental Data on the Disintegration of Liquid Jets," Industrial and Engineering Chemistry, Vol. 47, No. 9, 1955 , pp. 1690-1697.

${ }^{14}$ Ranz, W. E., "Some Experiments on Orifice Sprays," Canadian Journal of Chemical Engineering, Vol. 36, Aug. 1958, pp. 175-181.

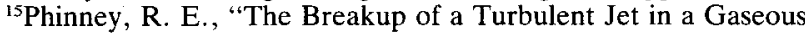
Atmosphere," Journal of Fluid Mechanics, Vol. 6, Pt. 4, Oct. 1973, pp. $689-701$

${ }^{16}$ Hoyt, J. W., and Taylor, J. J., "Waves on Water Jets," Journal of Fluid Mechanics, Vol. 88, Pt. 1, Nov. 1977, pp. 119-127.

${ }^{17}$ Hoyt, J. W., and Taylor, J. J., "Turbulence Structure in a Water Jet Discharging in Air," Physics of Fluids, Vol. 20, No. 10, Pt. II, 1977, pp. S253-S257.

${ }^{18}$ Hoyt, J. W., and Taylor, J. J., "Effect of Nozzle Boundary Layer on Water Jets Discharging in Air," Jets and Cavities, edited by J. H. Kim, O. Furuya, and B. R. Parkin, Vol. 31, ASME-FED, New York, 1985 , pp. 93-100.

${ }^{19}$ Hiroyasu, H., Shimizu, M., and Arai, M., "The Breakup of a 
High Speed Jet in a High Pressure Gaseous Environment," Univ. of Wisconsin, ICLASS-82, Madison, WI, 1982.

${ }^{20}$ Chehroudi, B., Onuma, Y., Chen, S.-H., and Bracco, F. V. "On the Intact Core of Full Cone Sprays," SAE Paper 850126, 1985.

${ }^{21}$ Wu, K.-J., Su, C.-C., Steinberger, R. L., Santavicca, D. A., and Bracco, F. V., "Measurements of the Spray Angle of Atomizing Jets," Journal of Fluids Engineering, Vol. 105, No. 4, 1983, pp. 406415 .

${ }^{22}$ Wu, K.-J., Coghe, A., Santavicca, D. A., and Bracco, F. V., "LDV Measurements of Drop Velocity in Diesel-Type Sprays," $A I A A$ Journal, Vol. 22, No. 9, 1984, pp. 1263-1270.

${ }^{23}$ Tseng, L.-K. " "Near-Injector Structure of Non-Evaporating Pressure-Atomized Sprays at Various Ambient Densities," Ph.D. Dissertation, Univ. of Michigan, Ann Arbor, MI 1991.

${ }^{24}$ Smith, R. H., and Wang, C.-T., "Contracting Cones Giving Uniform Throat Speeds," Journal of Aerospace Sciences, Vol. 11, No. 4, Oct. 1944, pp. 356-360.

${ }^{25}$ Schlichting, H., Boundary Layer Theory, 7th ed., McGraw-Hill, New York, 1979, p. 599.

${ }^{26}$ Hinze, J. O., Turbulence, 2nd ed., McGraw-Hill, New York, 1975, pp. 427, 724-734.
${ }^{27}$ Lockwood, F. C., and Naguib, A. S., "The Prediction of Fluctuations in the Properties of Free, Round-Jet Turbulent Diffusion Flames," Combustion and Flame, Vol. 24, No. 1, 1975, pp. 109-124.

${ }^{28}$ Bilger, R. W., "Turbulent Jet Diffusion Flames," Progress in Energy and Combustion Science, Vol. 1, No. 2, 1976, pp. 87-109.

${ }^{29}$ Simmons, H. C., "The Correlation of Drop Size Distributions in Fuel Nozzle Sprays," Journal of Engineering for Power, Vol. 99, No. 3, 1977, pp. 309-319.

${ }^{30} \mathrm{Krzeczkowski}$, S. A., "Measurement of Liquid Droplet Disintegration Mechanisms," International Journal of Multiphase Flow, Vol. 6, No. 2, 1980, pp. 227-239.

${ }^{31}$ Hsiang, L.-P., and Faeth, G. M., "Secondary Drop Breakup in the Deformation Regime," AIAA Paper 92-0110, 1992.

${ }^{32}$ Pruppacker, H. R., and Klett, J. D., Microphysics of Clouds and Precipitation, D. Reidel Publishing Co., Boston, MA, 1978, pp. 123456.

${ }^{33}$ Borisov, A. A., Gel'fand, B. E., Natanzon, M. S., and Kossov, O. M., "Droplet Breakup Regimes and Criteria for Their Existence,". Inzhenerno-Fizicheskii Zhurnal, Vol. 40, Jan. 1981, pp. 64-70.

${ }^{34}$ Ranger, A. A., and Nicholls, J. A., "Aerodynamic Shattering of Liquid Drops," AIAA Journal, Vol. 7, No. 2, 1969, pp. 285-290.

\section{A Best-Selling Trilogy 拳 AIAA Education Series}

\section{AEROTHERMODYNAMICS OF AIRCRAFT ENGINE COMPONENTS}

Gordon C. Oates, editor

Major topics include turbine cooling, boundary layer analysis in rotating machinery, engine noise, combustion, and afterburners.

1985, $551 \mathrm{pp}$, illus, Hardback

ISBN 0-915928-97-3

AIAA Members $\$ 46.95$

Nonmembers $\$ 57.95$

Order \#: 97-3 (830)

Place your order today! Call 1-800/682-AlAA UAMA

American Institute of Aeronautics and Astronautics Publications Customer Service, 9 Jay Gould Ct., P.O. Box 753, Waldori, MD 20604 Phone 301/645-5643, Dept. 415, FAX 301/843-0159 British Columbia

\section{AIRCRAFT PROPULSION SYSTEMS TECHNOLOGY AND DESIGN}

Gordon C. Oates, editor

“.... a practical reference text....excellent survey of important matters useful in aircraft design." -I. S. Gartshore, University of

A comprehensive coverage of the key physical concepts that govern gas turbine propulsion systems. Topics include combustion technology, engine/airplane performance matching, inlets and inlet/engine integration, variable convergent/divergent nozzle aerodynamics, and more.

1989,528 pp, illus, Hardback ISBN 0-930403-24-X

AIAA Members $\$ 46.95$

Nonmembers $\$ 57.95$

Order \#: 24-X (830)

\section{AEROTHERMODYNAMICS OF GAS TURBINE AND ROCKET PROPULSION: REVISED AND ENLARGED}

Gordon C. Oates

Contents include: thermodynamics and quasi-one-dimensional fluid flows; chemical and nonchemical rockets; ideal and nonideal cycle analysis; component performance; engine off-design performance; blade aerodynamics; SAE Gas Turbine Engine Notation appendices; and more.

1988, 452 pp, illus, Hardback

ISBN 0-930403-34-7

AIAA Members $\$ 46.95$

Nonmembers $\$ 57.95$

Order \#: 34-7 (830)

Sales Tax: CA residents, $8.25 \%$; DC, $6 \%$. For shipping and handling add $\$ 4.75$ for $1-4$ books (call for rates for higher quantities). Orders under $\$ 50.00$ must be prepaid. Please allow 4 weeks for delivery. Prices are subject to change without notice. Returns will be accepted within 15 days. 